\title{
Gold Nanoparticles Sliding on Recyclable Nanohoodoos-Engineered for Surface- Enhanced Raman Spectroscopy
}

\author{
Wu, Kaiyu; Li, Tao; Schmidt, Michael Stenbæk; Rindzevicius, Tomas; Boisen, Anja; Ndoni, Sokol
}

Published in:

Advanced Functional Materials

Link to article, DOI:

10.1002/adfm.201704818

Publication date:

2018

Document Version

Peer reviewed version

Link back to DTU Orbit

Citation $(A P A)$ :

Wu, K., Li, T., Schmidt, M. S., Rindzevicius, T., Boisen, A., \& Ndoni, S. (2018). Gold Nanoparticles Sliding on Recyclable Nanohoodoos-Engineered for Surface-Enhanced Raman Spectroscopy. Advanced Functional Materials, 28(2), [1704818]. https://doi.org/10.1002/adfm.201704818

\section{General rights}

Copyright and moral rights for the publications made accessible in the public portal are retained by the authors and/or other copyright owners and it is a condition of accessing publications that users recognise and abide by the legal requirements associated with these rights.

- Users may download and print one copy of any publication from the public portal for the purpose of private study or research.

- You may not further distribute the material or use it for any profit-making activity or commercial gain

- You may freely distribute the URL identifying the publication in the public portal 
DOI: $10.1002 /$

Article type: Full Paper

Gold Nanoparticles Sliding on Recyclable Nanohoodoos - Engineered for Surfaceenhanced Raman Spectroscopy

Kaiyu $\mathrm{Wu}^{\dagger}$, Tao Lit,*, Michael Stenboek Schmidt, Tomas Rindzevicius, Anja Boisen* and Sokol Ndoni

${ }^{\dagger}$ Equal contribution

(K.W. and T.L. contributed equally to this work. T.L. conceived and fabricated the prototype of the substrate. K.W. and T.L. conceived the further development of the substrate. K.W. performed the SERS measurements. M.S. suggested the recycling of the nanohoodoos. K.W. and T.L. realized the recycling of the nanohoodoos. K.W. performed the FEM simulations and the dark-field scattering measurements. T.L. did the SEM and the XPS characterizations. K.W. wrote the manuscript. All authors participated in the discussion of the results and improved the manuscript.)

Dr. K. Wu ${ }^{1}$, Assoc. Prof. M. S. Schmidt ${ }^{1}$, Assoc. Prof. T. Rindzevicius ${ }^{1}$, Prof. A. Boisen ${ }^{1}$ and Snr. Scientist S. Ndoni ${ }^{2}$

Department of Micro- and Nanotechnology

Technical University of Denmark

Ørsteds Plads, Building 345B, 2800 Kgs. Lyngby

Denmark

${ }^{1}$ DNRF and Villum Fonden Center for Intelligent Drug Delivery and Sensing Using

Microcontainers and Nanomechanics, IDUN, Ørsteds Plads, Building 345C, 2800 Kgs.

Lyngby, Denmark

2 DNRF Center for Nanostructured Graphene, CNG, Ørsteds Plads, Building 345B, 2800 Kgs. Lyngby, Denmark

Dr. T. Li

Department of Electronic \& Electrical Engineering

University College London

Torrington Place, London WC1E 7JE

UK

E-mail: tao.li@ucl.ac.uk, anja.boisen@nanotech.dtu.dk

Keywords: surface-enhanced Raman spectroscopy, SERS substrate, block copolymer lithography, recyclable, large-area SERS uniformity

Abstract Text:

Robust, macroscopically uniform, and highly sensitive substrates for surface-enhanced

Raman spectroscopy (SERS) are fabricated using wafer-scale block copolymer lithography.

The substrate consists of gold nanoparticles that can slide and aggregate on dense and recyclable alumina/silicon nanohoodoos. Hot spot engineering is conducted to maximize the 
SERS performance of the substrate. The substrate demonstrates remarkably large surfaceaveraged SERS enhancements, greater than $10^{7}\left(>10^{8}\right.$ in hot spots), with unrivalled macroscopic signal uniformity as characterized by a coefficient of variation of only $6 \%$ across $4 \mathrm{~cm}$. After SERS analyses, the nanohoodoos can be recycled by complete removal of gold via a one-step, simple and robust wet etching process without compromising performance. After 8 times of recycling, the substrate still exhibits identical SERS perfomance in comparison to a new substrate. The macroscopic uniformity combined with recyclability at conserved high performance is expected to contribute significantly on the overall competitivity of the substrates. Our findings show that the GNRH substrate is a very strong candidate for obtaining cost effective, high quality, and reliable SERS spectra, facilitating a wide and simple use of SERS for both laboratorial and commercial applications.

\section{Main Text:}

Surface-enhanced Raman spectroscopy (SERS) probes the significantly amplified Raman scattering signals from analytes attached to, or in the very close vicinity of metallic nanostructures. It allows ultrasensitive identification of a variety of analytes ranging from biomarkers to chemical warfare agents and toxic food additives. ${ }^{[1-3]}$ The field of SERS has attracted increased interest, and has expanded dramatically since its recognition in the $1970 \mathrm{~s},{ }^{[4-6]}$ thanks to the development of highly efficient laser systems, the rapid advances of nanotechnology, and the observation of SERS signals from single molecules. ${ }^{[7,8]}$ In the year 2016 alone, more than 1500 research articles regarding SERS have been published, ${ }^{[9]}$ demonstrating the power of SERS as an ultrasensitive molecular probing technique, and its value in a wide range of practical applications.

Essentially, SERS is a prominent spectroscopic application of noble metallic nanostructures which support localized surface plasmon resonances (LSPRs). In SERS, the vast majority of 
the enhanced Raman signal is obtained from the nanoscale electromagnetic (EM) hot spots, which are formed in the close vicinity of the metallic surfaces by couplings between free space photons and LSPRs. ${ }^{[10]}$ In an EM hot spot, both the incident field and the Raman scattering from molecules are amplified, leading to a total SERS intensity that scales with the forth power of the surface-averaged electrical field, i.e., $\mathrm{I}_{\mathrm{SERS}} \propto\left\langle\mathrm{E}^{4} / \mathrm{E}_{0}{ }^{4}>\right.$, where $\mathrm{E}_{0}$ is the incident field. ${ }^{[11]}$ To achieve a high SERS enhancement factor (EF), a hot spot that contains intense electrical field is required. This can be realized by metallic nanostructures with a small local radius of curvature. ${ }^{[12]}$ Recently, via accurate single hotspot engineering, SERS EFs of above $10^{10}$ have been achieved using nanodumbbells ${ }^{[13]}$ and nanostars ${ }^{[14]}$, enabling the detection of single non-resonant molecules.

The development of solid SERS substrates has been a hot topic in the field, and has attracted significant attention, as the successful development of SERS applications relies on the availability of highly enhancing, uniform and reproducible SERS substrates. ${ }^{[15]}$ To achieve widespread and reliable use in practical applications, the ideal SERS substrate should exhibit (i) high and reproducible surface-averaged EFs $>10^{7}$ over macroscopic areas (in inch scales), (ii) compatibility with large-format and high-volume manufacturing process flows with excellent batch-to-batch reproducibility, (iii) low manufacturing cost, and (iv) broad suitability and extended shelf life, i.e., long-term LSPR activity and stability.

Modern solid SERS substrates can be classified into four categories: (i) lithographically patterned substrates, using techniques such as laser interference lithography, ${ }^{[15]}$ nanoimprint lithography, ${ }^{[16]}$ and colloidal hole-mask lithography, ${ }^{[17]}$ (ii) patterned substrates using lithography-free techniques, such as mask-less reactive ion etching (RIE), ${ }^{[18]}$ (iii) structured film based substrates, e.g., noble metal on a rose petal, ${ }^{[19]}$ as well as nanoparticle clusters on slippery liquid-infused porous surfaces ${ }^{[20]}$, and (iv) particle supported substrates, e.g., immobilized nanorods on nanosphere assemblies ${ }^{[21]}$ as well as $3 \mathrm{D}$ cross-point plasmonic nanostructures fabricated by multistep nanotransfer printing. ${ }^{[22]}$ 
From a practical point of view, large-scale manufacturing of SERS substrates that require optical lithography patterning or complex synthesis is, in general, more costly than those that do not. ${ }^{[23]}$ State-of-the-art SERS substrates include nanocube-based plasmene nanosheets, which are fabricated by a scalable self-assembly process. ${ }^{[24,25]}$ One of the most cost-effective ways to fabricate efficient SERS substrates is the deposition of silver nanoparticles onto rose petals. ${ }^{[26]}$ However, the fabricated substrate may suffer from a lack of uniformity of the plasmonic nanostructures caused by the irregular and varying morphologies across the template.

To facilitate the use of SERS substrates in both laboratories and mobile applications, there is a need to narrow the gap between cost and excellent SERS performance, and improve shelflife which is often an issue ${ }^{[27]}$. A solution may be provided by metallic nanostructures on prepatterned reusable templates. After SERS analyses, the templates are recycled by the removal of the metallic nanostructures, followed by re-metallization to renew the substrates for the next SERS analyses. As a result, the cost will be minimized, since recycling the template and re-metallization are far simpler, faster, and more cost-effective than fabricating a new SERS substrate.

Although this approach sounds intriguing, some challenges remain. Firstly, the template should be robust enough to survive a recycling process. SERS substrates supported by mechanically or chemically unstable structures, e.g., nanoparticle templates ${ }^{[21]}$ and leaning nanopillars, ${ }^{[28]}$ are unsuitable. Secondly, dense and uniform hot spots with significantly enhanced electrical field must be achieved through well controlled hotspot engineering, to ensure high and reproducible surface-averaged EFs $>10^{7}$ over macroscopic areas (across centimeters). ${ }^{[29]}$ In addition, an auxiliary requirement is that the manufacturing process of the template is compatible with large-format and high-volume process flows.

Herein, we report gold nanoparticles sliding on recyclable nanohoodoos (GNRH) as SERS substrates that might prove advantageous with respect to all the expected properties of an 
ideal SERS substrate. Large-area, uniform and robust nanohoodoos are fabricated in waferscale using block copolymer (BCP) lithography, which constitutes a platform for nanofabrication with high resolution, high precision of control and high throughput. ${ }^{[30,31]}$ Gold is evaporated onto the nanohoodoos forming gold nanoparticles, creating a GNRH SERS substrate that can be used for both gas and liquid sensing. Gold is chosen due to its favorable chemical properties towards a wide range of applications and long-term plasmonic stabilities. Upon drying of a deposited analyte solution, the gold nanoparticles slide on the nanohoodoos and form particle clusters with strong, dense and uniform EM hot spots. Moreover, after SERS analyses, the nanohoodoos can be recycled by complete removal of gold via wet etching. The reused substrate exhibits reproducibly low SERS backgrounds as well as identical SERS perfomance in comparison to a new substrate, which lowers the cost of the GNRH SERS substrate. Concerning SERS performance, hot spots of the GNRH substrate are carefully engineered according to finite element method (FEM) simulations, and are maximized exprimentally for excitation at $780 \mathrm{~nm}$. The developed substrate demonstrates a remarkably large surface-averaged SERS EF of $\sim 1.1 \times 10^{7}$, with unrivalled macroscale uniformity of $\sim 6 \%$ coefficient of variation (CV) across $4 \mathrm{~cm}$.

The fabrication of nanohoodoos is schematically illustrated in Figure 1a. Using BCP lithography, a 2-inch silicon wafer is first patterned by highly ordered nanoholes. The details of this process can be found in the references. ${ }^{[32,33]}$ Then, $26 \mathrm{~nm}$ of alumina is deposited onto the nanoholes using atomic layer deposition (ALD). ALD is chosen since it deposits alumina conformably onto the pre-patterned substrate. As a result, all the nanoholes are seamlessly filled by alumina, part of which will be used later as an etching mask. Additionally, ALD is a standard high-volume manufacturing technique in the IC industry, employed widely for fabrication of numerous components. ${ }^{[34]}$ The ALD process is followed by chlorine-based reactive ion etching (RIE). During RIE, after the breakthrough of the top continuous alumina layer, the silicon domain is etched much faster than the alumina domain. The remaining 
alumina in the nanoholes then serves as a hard mask during the following silicon etching. ${ }^{[35]}$ This process reverses the pattern relative to the initial mask, rendering well ordered, hexagonally packed alumina topped silicon nanocylinder arrays over the wafer. These nanocylinders are visualized using SEM from a cross-sectional view in Figure 1b, and from a vertical view in Figure S1a (see supporting information). Next, hierarchical nanohoodoos consisting of silicon nanospikes with alumina disks, as displayed in Figure 1c, are realized by deliberately introducing fluorine-based RIE to the chlorine-based RIE. A macroscopic picture of a 2-inch silicon wafer covered with arrays of these nanohoodoos is shown in Figure 1e. Silicon etching using chlorine-based RIE is highly anisotropic, whereas silicon etching using fluorine-based RIE in this case is intentionally isotropic. ${ }^{[36]}$ The isotropic etch turns the alumina/silicon nanocylinders into alumina/silicon nanohoodoos, as the alumina layer is highly resistant towards fluorine-based RIE. ${ }^{[36]}$ Finally, a layer of gold is deposited onto the nanohoodoos by electron-beam evaporation. This process renders a layer of separated gold nanoparticles on top of the alumina disks, as is shown in Figure 1d. Note that the resulting gold nanoparticles possess a very high density $\left(\sim 140 / \mu \mathrm{m}^{2}\right)$, which is needed to achieve numerous nanoparticle clusters that contains a large number of SERS hot spots. ${ }^{[37]} \mathrm{A}$ photograph of a wafer-scale (2-inch) GNRH substrate is shown in Figure 1f.

The electromagnetic enhancement arising from the hot spots is the major and decisive contributor to the SERS effect. ${ }^{[38]}$ In plasmonic structures without sharp metallic tips, hot spots are generally realized via the formation of nanogaps between metallic structures. ${ }^{[39]}$ On the GNRH substrate, the gold nanoparticles are able to detach themselves from the nanohoodoos by the surface tension occurring upon drying of solvent, owing to the poor adhesion between gold and alumina. ${ }^{[40]}$ Subsequently, gold nanoparticles slide off and form particle clusters of densely packed particles in a two dimensional geometry that efficiently generates EM hot spots. Evidence showing the detachment and aggregation of the gold nanoparticles are provided by the SEM images in Figures 2d - f. It is essential that the 
nanohoodoos have a uniform height so that the nanoparticles are able to "find" their nearest neighbors when aggregating.

The nanohoodoo design is crucial for obtaining movable gold nanoparticles. If vertical nanocylinders, fabricated using only chlorine-based RIE, are used as template, the resulting metallization would cover the side walls of the nanocylinders and are thus rendering the formed nanoparticles immobile, as is shown in Figure $\mathbf{S 2}$ in the supporting information. These structures are significantly less SERS-active due to their inability to form gold nanoparticle aggregates with small gap junctions.

The GNRH SERS substrate is capable of sensing analytes in both gas and liquid phases. The schematic of gas sensing is shown in Figures $2 \mathrm{a}-\mathrm{c}$. When the substrate is exposed to analytes in gas phase, the analytes adsorb onto the gold nanoparticles on the nanohoodoos. After formation of nanoparticle aggregations via depositing and drying of a pure solvent, those analytes that sit inside or near hot spots, i.e., gap junctions between the gold nanoparticles, radiate strongly enhanced Raman scattering signals and are thus detectable. In a slightly different way, to sense analytes in liquid, a solvent containing analytes is deposited onto the substrate. In this case, the adsorption of the analytes to the gold nanoparticles takes place on a wet substrate. Alternatively, the substrate can be immersed in the analyte solution for incubation. Nanoparticles then slide and form clusters during the drying of the substrate.

To prove and emphasize that the detachment and clustering of the gold nanoparticles is essential for obtaining strong SERS signals, the following experiments are performed. The GNRH substrate is placed in the headspace above a $1 \mathrm{mM}$ thiophenol in ethanol solution for 1 minute. Afterwards, clusters of gold nanoparticles are formed via deposition and evaporation of DI water. SERS measurements are performed on the substrate before and after deposition and drying of DI water. Results are shown in Figure $2 \mathrm{~h}$. The SERS signal obtained on the nanohoodoos covered by the gold nanoparticle clusters is approximately 30 times higher than that obtained on the substrate before deposition of DI water. Such a high signal increase is 
expected, as the SERS intensity scales with the fourth power of the magnitude of the local electrical field, which is significantly enhanced when individual nanoparticles form aggregates. ${ }^{[11]}$ In other words, the detachment and clustering of the gold nanoparticles is essential for the formation of strong SERS hot spots with significantly enhanced electromagnetic fields. Therefore, in practical use, to achieve optimal SERS signals on a GNRH substrate, solvent needs to be deposited onto the substrate and evaporate, to ensure that all gold nanoparticles are detached from the nanohoodoos, so that maximum numbers of nanoparticle aggregates are formed.

The far-field optical response of the GNRH SERS substrate is presented in Figure $2 \mathrm{~g}$ by the dark-field scattering spectra. For the measured substrate, a gold deposition of $40 \mathrm{~nm}$ is employed, since it gives the best SERS performance at $780 \mathrm{~nm}$ excitation. A $40 \mathrm{~nm}$ deposition also prevents the rendered gold nanoparticles $\left(\sim 140\right.$ nanoparticles $\left./ \mu \mathrm{m}^{2}\right)$ from linking to their neighbors. In addition, the rendered gold nanoparticles are $\sim 30 \mathrm{~nm}$ in radius. It can be seen from Figure $2 \mathrm{~g}$ that, after clustering of the gold nanoparticles, the scattering peak maximum red shifts from $\sim 700 \mathrm{~nm}$ to $\sim 800 \mathrm{~nm}$. This red shift can be attributed to the collective effect of the strong plasmonic couplings among the aggregated gold nanoparticles. Additionally, broad scattering peaks covering a spectral range from $\sim 650$ to $\sim 850 \mathrm{~nm}$ are observed for both the nanohoodoos topped by separate gold nanoparticles and gold nanoparticle aggregations. Such broad peaks can be attributed to (i) the variation in size and shape of the gold nanoparticles, ${ }^{[41]}$ (ii) the uneven distance between the adjacent gold nanoparticles, and (iii) different configurations of the nanoparticle aggregates. ${ }^{[42]}$

3D FEM simulations are performed to theoretically predict the locations of the hot spots as well as the maximum SERS EF for the GNRH SERS substrate. For simplicity, a hexagonal array consisting of seven nanohoodoos each topped by a gold nanosphere is considered. The simulated geometries are shown in Figures 3a and 3b, for the nanohoodoos topped by separate and aggregated gold nanoparticles, respectively. The electrical field enhancement 
factor (EEF), i.e., $\mathrm{E} / \mathrm{E}_{0}$, where $\mathrm{E}_{0}$ is the magnitude of the incident field, is calculated throughout the solution domain. Upon clustering of the gold nanoparticles, it is found that the hot spots are located at the gap junctions between the gold nanoparticles, as is illustrated by the electrical field distribution shown in Figure 3e. In addition, with a minimum gap distance between the two adjacent gold nanoparticles being $1 \mathrm{~nm}$, the calculated maximum EEF is 121 , which, according to the $\mathrm{E}^{4}$ approximation, leads to a maximum electromagnetic SERS enhancement factor of $\sim 2.1 \times 10^{8}$.

A high EEF is a prerequisite for a plasmonic system to be used for efficient SERS analyses. It is found that the height of the nanostructures on the GNRH substrate has a distinct influence on the intensity of the SERS signals. Here, the height $h$ is defined as the vertical distance from the center of the gold nanoparticle layer to the underlying gold film on the silicon substrate, as is visualized in the inset of Figure $3 \mathrm{~b}$. By tuning $h$, the gold nanoparticle aggregation on top of the nanohoodoos experiences an excitation field with modulated intensity, due to the interference of the incident and reflected fields at the bottom of the substrate, which can be regarded as a mirror. This effect can be used to strengthen the intensity of the LSPRs of the metallic nanostructures above a substrate, leading to stronger near fields and thus stronger SERS signals. ${ }^{[43]}$ In theory, for reflection of an electromagnetic wave at a conducting surface, at normal incidence, the scalar of the total field in the incident medium can be expressed as $E$ $=2 E_{0}(\sin \omega t)(\sin k x)$, where $x$ is the vertical distance from the incident medium to the interface. ${ }^{[44]}$ The field reaches its maximum when $\sin k x=1$. Assuming that the incident medium is air $(n=1)$, to get $x$ for the first constructive interference pattern, $k x=2 \pi x / \lambda=\pi / 2$, leading to $x=4 / \lambda$. Therefore, for SERS measurement using $780 \mathrm{~nm}$ excitation, the optimal height $h$ for the GNRH substrate should be around a quarter of the excitation wavelength, i.e., $\sim 195 \mathrm{~nm}$. To demonstrate such an effect for the GNRH substrate, additional FEM simulation is performed for an aggregation of gold nanoparticles placed directly on a silicon substrate, as shown by Figure 3c. The calculated EEF is shown in Figure $3 \mathrm{f}$. A maximum EEF of 78 is 
obtained. Using the $\mathrm{E}^{4}$ approximation, this leads to an estimated maximum electromagnetic SERS enhancement factor of $\sim 3.7 \times 10^{7}$, which is $\sim 6$ times lower than that estimated for the GNRH SERS substrate with $h=190 \mathrm{~nm}$, shown in Figure 3e.

Following the strategy and results provided by the simulations, $h$ is experimentally optimized for the GNRH substrate to maximize its SERS EF for $780 \mathrm{~nm}$ excitations. $h$ is changed by varying the process time of the mixed chlorine- and fluorine-based RIE. To demonstrate the optimization, averaged SERS spectra of 1 nmol trans-1,2-bis (4-pyridyl) ethylene (BPE) in ethanol solution are recorded on the substrates with $h$ of $\sim 160, \sim 190$, and $\sim 220 \mathrm{~nm}$, shown correspondingly in Figure 3h-j. At $h=190 \mathrm{~nm}(\sim 1 / 4$ of the excitation wavelength), the gold nanoparticles are expected to be "sitting" near the strongest constructive interference pattern of the excitation field. This should strengthen the average intensity of the electrical fields in the hot spots, and the molecules should therefore radiate the strongest SERS signals. Indeed, as is seen from Figure 3g, the strongest SERS signals are obtained on the substrate with $h=190 \mathrm{~nm}$.

The SERS EF of the GNRH substrate is estimated experimentally using the following equation:

$$
\mathrm{EF}=\frac{\mathrm{I}_{\mathrm{SERS}} / \mathrm{N}_{\mathrm{SERS}}}{\mathrm{I}_{\mathrm{RS}} / \mathrm{N}_{\mathrm{RS}}}
$$

where $I_{S E R S}$ and $I_{R S}$ are the SERS and the Raman intensity, respectively. $N_{\text {SERS }}$ and $N_{R S}$ are the numbers of molecules probed in the SERS and the Raman measurements. To establish the values of $\mathrm{I}_{R S}$ and $\mathrm{N}_{\mathrm{RS}}$, a $0.1 \mathrm{M}$ ethanol solution of trans-1,2-Bis(4-pyridyl)ethylene (BPE) is poured into a petri dish. 1.4 counts $\mathrm{mW}^{-1} \cdot \mathrm{s}^{-1}\left(\mathrm{I}_{\mathrm{RS}}\right)$ are measured for the $1200 \mathrm{~cm}^{-1}$ peak under an excitation wavelength of $780 \mathrm{~nm}$. The number of molecules excited $\left(\mathrm{N}_{\mathrm{RS}}\right)$ is estimated to be $3.60 \times 10^{9}$, using the interaction volume of $60 \mu \mathrm{m}^{3}$. To establish the values for ISERS and $\mathrm{N}_{\mathrm{SERS}}, 1 \mathrm{nmol} \mathrm{BPE}$ in ethanol solution is pipetted onto the GNRH substrate. The droplet quickly spreads over the substrate surface, and dries within a few seconds. The introduced 
BPE solution forms a quasi-circular pattern on the surface of the substrate with a radius of $\sim 3500 \mu \mathrm{m}$. Assuming a uniform distribution, this leads to a density of BPE on the substrate to be $1.56 \times 10^{7} / \mu \mathrm{m}^{2}$. Using identical conditions as for $\mathrm{I}_{\mathrm{RS}}, \sim 30000 \mathrm{~mW}^{-1} \cdot \mathrm{s}^{-1}\left(\mathrm{I}_{\mathrm{SERS}}\right)$ are measured for the $1200 \mathrm{~cm}^{-1}$ peak. Assuming uniform adsorption of BPE onto gold, and taking the topography of the gold nanoparticles and film into consideration, the number of adsorbed BPE molecules per nanoparticle is estimated to be 24800 . With a density of $\sim 140$ nanoparticles $/ \mu \mathrm{m}^{2}$ and a laser spot size of $0.8 \mu \mathrm{m}$ in radius, $\mathrm{N}_{\mathrm{SERS}}$ is estimated to be $7 \times 10^{6}$. Hence, the calculated surface-averaged EF becomes $1.1 \times 10^{7}$. Note that this EF is an averaged value, experimentally obtained whilst assuming that all the adsorbed molecules contribute equally to the integrated SERS signal, ${ }^{[45]}$ and is thus not the maximum enhancement which others have attempted to calculate by assuming that all of the signal derives from a much lower number of molecules only located in the hot spots.

The primary function of a SERS substrate is to enhance Raman signals of trace amount of analytes to a detectable level. To demonstrate this for the GNRH substrate, BPE molecules of different quantities from $10^{-8} \mathrm{~mol}$ to $10^{-12} \mathrm{~mol}$, in ethanol, are deposited onto the substrates. After drying of the substrates in air, SERS responses are measured. The corresponding spectra are shown in Figure 4a. It can be seen from Figure 4a that trace amount BPE of down to 1 pmol can be detected. Such results imply that the GNRH substrate is a very capable candidate for extensive SERS applications.

There has been an increasing emphasis on the reproducibility and uniformity of the EF to ensure consistent chemical detection sensitivity across the surface of a SERS substrate. This is because large-area SERS enhancement uniformity is a fundamental prerequisite to achieve reliable measurements, and a prerequisite if quantitative SERS is to be realized. ${ }^{[46]}$ It is necessary to point out that the term "large-area" can be somewhat misleading. In some parts of the literature, good SERS uniformities over tens to hundreds of $\mu \mathrm{m}$ are labelled "large- 
area". In this work, the term macroscale is used, and the SERS uniformity in $\mathrm{mm}$ and $\mathrm{cm}$ scales is demonstrated for the GNRH substrate.

To evaluate the reproducibility of the SERS sensitivity across the GNRH substrate, 1 pmol BPE in ethanol is deposited onto the substrate. SERS mappings are conducted after solvent evaporation. SERS mapping of the full droplet area with a step size of $100 \mu \mathrm{m}$ is shown in Figure $4 \mathrm{~b}$. It can be seen that the droplet occupies a quasi-circular area of $\sim 5 \mathrm{~mm}$ in radius. Additionally, the intensity of the signals in the inner area with a $\sim 3 \mathrm{~mm}$ radius is $\sim 3$ times higher than in the outer area. Furthermore, the signals are more uniform near the center of the droplet. The symmetry of the intensity map towards the center of the droplet $(\sim 5 \mathrm{~mm}$ in radius), indicates that the GNRH substrate is capable of reproducing SERS spectra in $\mathrm{mm}$ scale. To further investigate the SERS enhancing reproducibility of the substrate, SERS mappings are carried out over a $1 \mathrm{~mm} \times 1 \mathrm{~mm}$ and a $100 \mu \mathrm{m} \times 100 \mu \mathrm{m}$ square near the center of the droplet, with a step size of $10 \mu \mathrm{m}$ and $5 \mu \mathrm{m}$, respectively. Results are shown in Figure 4c-d. To achieve quantitative analysis, CVs of the $1609 \mathrm{~cm}^{-1}$ BPE SERS peak are calculated for both cases. $\sim 20 \%$ and $\sim 10 \%$ are obtained over areas of $1 \mathrm{~mm} \times 1 \mathrm{~mm}$ and $100 \mu \mathrm{m} \times 100$ $\mu \mathrm{m}$, respectively. Such results demonstrate that the GNRH substrate is able to enhance Raman spectra for analytes of extremely small quantities, in this case 1 pmol BPE, in a reproducible and reliable manner.

To study and explain the rather uniform enhancement of the GNRH SERS substrate, additional 3D FEM simulations are conducted. The simulated geometry is the same as is in Figure 3a, except for that the nanohoodoos are topped by gold nanoparticle clusters of different configurations. Note that practically, the aggregating of the gold nanoparticles is random, thus it is not feasible to simulate all the situations. Therefore, clusters of 12 different configurations, from a dimer to a hexagonal array, are simulated as representatives. EEF is calculated throughout the solution domain, under excitation of two perpendicularly polarized plane waves at $780 \mathrm{~nm}$. To visualize, Figure 5a shows the calculated EEF across the central 
plane of the gold nanoparticle layer. To quantify and relate these results to hot-spot uniformity, the averaged maximum EEF per nanogap is plotted against the configuration of the nanoparticle clusters, as is shown by Figure 5b. From Figure 5b, it can be concluded that, the average contribution per nanogap, i.e., per hotspot, to the electromagnetic EF is quite similar, despite the different configurations of the nanoparticle clusters. Therefore, the rather uniform enhancing behavior of the GNRH SERS substrate can be attributed to (i) the fairly uniform properties of the hot spots indicated by the simulation, and (ii) the averaging effect of the laser, caused by simultaneously probing a great many of hotspots (the density of the gold nanoparticles is $\sim 140 / \mu \mathrm{m}^{2}$ ), using a laser with a spot size of around several $\mu \mathrm{m}^{2}$.

Here, macroscopic uniformity on the $\mathrm{cm}$ scale is investigated across a 2-inch GNRH substrate. For this purpose, a SERS line scan across $4 \mathrm{~cm}$ is conducted on a BPE covered substrate. The results are shown in Figure 6b. The presented result indicates that the GNRH substrate exhibits unrivalled macroscale SERS uniformity. To quantify, CV is calculated for the intensity of the $1609 \mathrm{~cm}^{-1}$ SERS peak, based on the 41 data points with a step size of 100 $\mu \mathrm{m}$ shown in the inset of Figure $6 \mathrm{~b}$. A value of $\sim 6 \%$ is obtained. This result indicates that the developed process is capable of fabricating reproducible SERS-active structures across a 2inch silicon wafer. In addition, the excellent macroscopic uniformity ensures that the SERS EF is reproducible. These findings further suggest that the GNRH substrate is a very strong candidate for obtaining reproducible and reliable SERS spectra.

A fast, robust and simple process that enables customized metallization and reuse of the nanohoodoos was developed as shown in Figure 6a. After SERS analyses, the nanohoodoos are recycled by complete removal of gold via wet etching. The substrate is first immersed in a solution of $\mathrm{KI}: \mathrm{I}_{2}: \mathrm{H}_{2} \mathrm{O}(100 \mathrm{~g}: 25 \mathrm{~g}: 500 \mathrm{ml})$ for 3 minutes, followed by rinsing with DI water and ethanol. Since the nanohoodoos are mechanically and chemically robust, all of them survive the recycling process, as is indicated by Figure $\mathrm{S} 1 \mathrm{~b}$ in the supplementary information. In addition, Figure $\mathbf{S 3}$ in the supplementary information shows the XPS spectra obtained on 
both the GNRH substrate and the recycled nanohoodoos. Comparing the spectra, it can be seen that the gold peaks disappear completely in the spectrum obtained on the recycled nanohoodoos, indicating complete removal of gold by the simple wet etching process.

To regenerate the GNRH substrate, a fresh layer of gold is deposited onto the top of the bare nanohoodoos via e-beam evaporation, which is widely accessible in almost any nanofabrication facilities and is easy to operate. The regenerated substrate exhibits almost no SERS background across the $4 \mathrm{~cm}$ scanning distance, as shown by Figure 6c. Moreover, comparing the SERS line scans in Figures $6 \mathrm{~b}$ and $6 \mathrm{~d}$, it can be seen that the regenerated substrate displays identical SERS performance without any degradation, in comparison to the first gold deposition. To further examine the robustness of the regeneration process, a GNRH substrate is regenerated 8 times. It can be seen from Figure 7 that its SERS performance maintains the same level without degradation. In addition, the recycling of the template and re-metallization can be carried out shortly before the planned SERS analyses to minimize contamination of the metallic surface. This would solve the issue of limited shelf life of plasmonic substrates, and would maximize performance. Finally, the user has the possibility of exploring different metal coatings.

In conclusion, robust, macroscopically uniform and highly sensitive GNRH SERS substrates are fabricated using wafer-scale BCP lithography. The substrate demonstrates remarkably large surface-averaged SERS enhancements greater than $10^{7}\left(>10^{8}\right.$ in hot spots), along with unrivalled macroscopic signal uniformity of $6 \% \mathrm{CV}$ across $4 \mathrm{~cm}$. The reported GNRH substrate yields the highest surface-averaged SERS EF to date that is reproducible across a macroscopic distance $(4 \mathrm{~cm})$. Additionally, after SERS analyses, the nanohoodoos in the GNRH substrate are recycled by complete removal of gold via a one-step simple wet chemistry process. The regenerated substrate exhibits reproducibly low SERS backgrounds and identical SERS perfomance in comparison to a new substrate. The success of the simple, cheap and robust regeneration process minimizes the cost of the GNRH substrate and 
maximizes its shelf life. Our findings suggest that the GNRH substrate is a strong candidate for obtaining high quality and reliable SERS spectra at a fairly low cost. It therefore facilitates a wide and simple use of SERS for both laboratorial and commercial applications.

\section{Experimental Section}

Fabrication of GNRH substrate: The experimental details to manufacture silicon nanohole arrays can be found in references. ${ }^{[32,33]}$ The atomic layer deposition was conducted in Picosun ALD model R200, using alternating exposures to trimethylaluminum (97\%, Aldrich) and deionized water at $200{ }^{\circ} \mathrm{C}$ for 300 cycles with purge steps using nitrogen gas in between. The exposure and purge time for both precursors used in this study was $0.2 \mathrm{~s}$ and $25 \mathrm{~s}$, respectively. The RIE was performed in ICP Metal Etch (SPTS serial no. MP0637). First step: chlorinebased etching: $20 \mathrm{sccm}$ chlorine, $3 \mathrm{mTorr}, 300 \mathrm{~W}$ of coil power and $40 \mathrm{~W}$ of platen power for 3 minutes. Second step: mixed chlorine- and fluorine-based etching: $20 \mathrm{sccm}$ chlorine gas and $3 \mathrm{sccm}$ sulfur fluoride, $3 \mathrm{mTorr}, 300 \mathrm{~W}$ of coil power and $40 \mathrm{~W}$ of platen power for 30 seconds. E-beam evaporation of gold was conducted in Wordentec (QCL 800) at a pressure of $4 \times 10^{-6}$ mbar. The deposition rate was $5 \AA / \mathrm{s}$ and the thickness of the deposited gold layer varied by $\pm 3 \%$. The removal of gold was conducted by immersing the substrate into the solution with KI:I2:H2O (100g:25g:500ml) for 3 minutes, followed by washing with water and ethanol for 2 times.

SEM and XPS characterizations: SEM images were taken by a field emission Zeiss Ultra Plus scanning electron microscope with a Gemini column operating at an accelerating voltage of 2 $\mathrm{kV}$. The cross-sectional images were taken at a tilted angle of $70^{\circ}$. All the samples were imaged directly without coating or staining. XPS measurement is performed in a ThermoScientific K-alpha XPS system.

SERS Measurements: SERS system 1: SERS measurements were performed using a Thermo Scientific DXR Raman microscope. The spectra were collected using a 50× objective lens and an excitation wavelength of $780 \mathrm{~nm}$. A $25 \mu \mathrm{m}$ slit was used. SERS system 2: A Thermo 
Scientific DXRxi Raman microscope was used. a $780 \mathrm{~nm}$ laser was used for excitation. The scattered light was collected by a $5 \times$ objective lens (Figure 6 ), and a $10 \times$ objective (Figure 4 and 7). A $50 \mu \mathrm{m}$ slit was used. For both systems, the microscopes were coupled to a single grating spectrometer with a $5 \mathrm{~cm}^{-1} \mathrm{FWHM}$ spectral resolution and a \pm 2 wavenumber accuracy. All SERS measurements were conducted at room temperatures. The absolute Raman intensities of the instruments over their detectable spectral ranges were calibrated according to known lamp spectra along with the Raman signals obtained on solid polystyrene. Dark-field Scattering Measurements: A Nikon Ti-U inverted microscope was used with a CF LU Plan Fluor Epi 50× objective, which had a working distance of $1 \mathrm{~mm}$ and a NA of 0.8 . The incident angle was $\sim 50^{\circ}$. The scattered light was collected from the center of the objective and was subsequently guided into a spectrometer (Shamrock Spectrograph SR-303IA, equipped with an Andor Newton 970 EMCCD). The obtained scattering spectrum was then divided by the reference white light spectrum to obtain the scattering signal from the substrates.

FEM simulation: 3D FEM simulations were performed using COMSOL MULTIPHYSICS ver. 4.4. The electromagnetic field distribution was calculated by solving the full field. The excitation field was generated on a domain-backed port, placed at the top of the solution domain, and was linearly polarized. Its wavelength was $780 \mathrm{~nm}$. A perfect matched layer with scattering boundary conditions on its outer boundaries was employed to eliminate nonphysical reflections. The boundary conditions for the side planes of the solution domain were Floquet periodicity. Materials were modeled by their complex refractive indices as functions of wavelength. ${ }^{[47-49]}$ The medium surrounding the structures was set to vacuum.

\section{Supporting Information}

Supporting Information is available online from the Wiley Online Library or from the author. 
This work has been supported by the IDUN project, The Danish National Research Foundation, Project DNRF122 and Villum Fonden, Grant No. 9301, the HERMES project, the European Research Council, Grant No. 320535; the Center for Nanostructured Graphene sponsored by the Danish National Research Foundation, Project DNRF103; the NAPLAS project, The Danish Council for Independent Research; the Dept. of Micro and Nanotechnology at the Technical University of Denmark; and the Danish national center for micro- and nanofabrication at the Technical University of Denmark.

Received: ((will be filled in by the editorial staff))

Revised: ((will be filled in by the editorial staff))

Published online: ((will be filled in by the editorial staff))

\section{References}

[1] D. Graham, R. Van Duyne, B. Ren, Analyst, 2016, 141, 4995.

[2] A. Hakonen, P. O. Andersson, M. S. Schmidt, T. Rindzevicius, M. Käll, Analytica

Chimica Acta, 2015, 893, 1.

[3] A. P. Craig, A. S. Franca, J. Irudayaraj, Annu. Rev. Food Sci. Technol., 2013, 4, 369.

[4] D. L. Jeanmarie, R. P. Van Duyne, J. Electroanal. Chem., 1977, 84, 120.

[5] M. G. Albrecht, J. A. Creighton, J. Am. Chem. Soc., 1977, 99, 5215.

[6] M. Fleischmann, P. J. Hendra, A. J. McQuillan, Chem. Phys. Lett., 1974, 26, 163.

[7] S. Nie, S. R. Emory, Science, 1997, 275, 1102.

[8] K. Kneipp, Y. Wang, H. Kneipp, L. T. Perelman, I. Itzkan, R. R. Dasari, .M. S. Feld, Phys. Rev. Lett., 1997, 78, 1667.

[9] Thomson Reuters, Web of Science Core Collection.

[10] E. C. Le Ru, P. G. Etchegoin, Annu. Rev. Phys. Chem., 2012, 63, 65.

[11] K. A. Willets, R. P. Van Duyne, Annu. Rev. Phys. Chem., 2007, 58, 267.

[12] K. L. Wustholz, A-I. Henry, J. M. McMahon, R. G. Freeman, N. Valley, M. E. Piotti, M. J. Natan, G. C. Schatz, R. P. Van Duyne, J. Am. Chem. Soc., 2010, 132, 10903.

[13] D.-K. Lim, K.-S. Jeon, H. M. Kim, J.-M. Nam, Y. D. Suh, Nat. Mater., 2010, 9, 60. 
[14] L. Rodríguez-Lorenzo, R. A. Álvarez-Puebla, I. Pastoriza-Santos, S. Mazzucco, O.

Stéphan, M. Kociak, L. M. Liz-Marzán, F. J. García de Abajo, J. Am. Chem. Soc., 2009, 131, 4616.

[15] K. N. Kanipe, P. P. F. Chidester, G. D. Stucky, M. Moskovits, ACS Nano, 2016, 10, 7566.

[16] T. Ding, D. O. Sigle, L. O. Herrmann, D. Wolverson, J. J. Baumberg, ACS Appl. Mater. Interfaces, 2014, 6, 17358.

[17] A. Hakonen, M. Svedendahl, R. Ogier, Z.-J. Yang, K. Lodewijks, R. Verre, T. Shegai, P. O. Anderssonb, M. Käll, Nanoscale, 2015, 7, 9405.

[18] M. S. Schmidt, J. Hübner, A. Boisen, Adv. Mater., 2012, 24, OP11.

[19] B.-B. Xu, Y.-L. Zhang, W.-Y. Zhang, X.-Q. Liu, J.-N. Wang, X.-L. Zhang, D.-D.

Zhang, H.-B. Jiang, R. Zhang, H.-B. Sun, Adv. Optical Mater., 2013, 1, 56.

[20] S. Yang, X. Dai, B. B. Stogin, T.-S. Wong, Proc. Natl. Acad. Sci. USA, 2016, 113, 268.

[21] N. Greeneltch, M. Blaber, A-I. Henry, G. Schatz, R. P. Van Duyne, Anal. Chem., 2013, $85,2297$.

[22] J. W. Jeong, M. M. P. Arnob, K.-M. Baek, S. Y. Lee, W.-C. Shih, Y. S. Jung, Adv. Mater., 2016, 28, 8695.

[23] N. G. Greeneltch, M. G. Blaber, J. A. Dieringer, G. C. Schatz, R. P. Van Duyne, J. Phys. Chem. C., 2013, 117, 2554.

[24] K. J. Si, P. Guo, Q. Shi, W. Cheng, Anal. Chem., 2015, 87, 5263.

[25] Y. Chen, K. J. Si, D. Sikdar, Y. Tang, M. Premaratne, W. Cheng, Adv. Opt. Mat., 2015, 3,919 .

[26] S.-Y. Chou, C.-C. Yu, Y.-T. Yen, K.-T. Lin, H.-L. Chen, W.-F. Su, Anal. Chem., 2015, $87,6017$.

[27] N. Nuntawong, P. Eiamchai, B. Wong-ek, M. Horprathum, K. Limwichean, V. Patthanasettakul, P. Chindaudom, Vacuum, 2013, 88, 23. 
[28] K. Wu, T. Rindzevicius, M. S. Schmidt, K. B. Mogensen, A. Hakonen, A. Boisen, J. Phys. Chem. C, 2015, 119, 2053.

[29] B. Sharma, M. F. Cardinal, S. L. Kleinman, N. G. Greeneltch, R. R. Frontiera, M. G. Blaber, G. C. Schatz, R. P. Van Duyne, MRS Bulletin, 2013, 38, 615.

[30] C. M. Bates, M. J. Maher, D. W. Janes, C. J. Ellison, C. G. Willson, Macromolecules, 2014, 47, 2.

[31] J. Bang, U. Jeong, D. Y. Ryu, T. P. Russell, C. J. Hawker, Adv. Mater., 2009, 21, 4769.

[32] T. Li, Z. Wang, L. Shulte, O. Hansen, S. Ndoni, RSC Adv., 2015, 5, 102619.

[33] T. Li, Z. Wang, L. Schulte, S. Ndoni, Nanoscale, 2015, 8, 136.

[34] Picosun Oy, picosun.com, http://www.picosun.com/applications/integrated-circuits-ic/

[35] T. Li, K. Wu, T. Rindzevicius, Z. Wang, L. Schulte, M. S. Schmidt, A. Boisen, S.

Ndoni, ACS Appl. Mater. Interfaces, 2016, 8, 15668.

[36] K. R. Williams, K. Gupta, M. Wasilik, J. Microelectromech. Syst., 2003, 12, 761.

[37] N. Pazos-Perez, C. S. Wagner, J. M. Romo-Herrera, L. M. Liz-Marzán, F. J. García de Abajo, A. Wittemann, A. Fery, R. A. Alvarez-Puebla, Angew. Chem. Int. Ed., 2012, 51, 12688.

[38] M. Moskovits, Phys. Chem. Chem. Phys., 2013, 15, 5301.

[39] L. Tong, H. Xu, M. Käll, MRS Bulletin, 2014, 39, 163.

[40] J.-M. Moon, A, Wei, J. Phys. Chem. B, 2005, 109, 23336.

[41] X. Lu, M. Rycenga, S. E. Skrabalak, B. Wiley, Y. Xia, Annu. Rev. Phys. Chem., $2009,60,167$.

[42] X. Tian, Y. Zhou, S. Thota, S. Zou, J. Zhao, J. Phys. Chem. C, 2014, 118, 13801.

[43] K. Wu, T. Rindzevicius, M. S. Schmidt, K. B. Mogensen, S. Xiao, A. Boisen, Opt. Express, 2015, 23, 12965.

[44] W. H. Hayt, J. A. Buck, Engineering Electromagnetics: Sixth Edition, McGraw-Hill, NY, USA, 2001. 
[45] S. L. Kleinman, B. Sharma, M. Blaber, A-I. Henry, N. Valley, R. G. Freeman, M. J.

Natan, G. C. Schatz, R. P. Van Duyne, J. Am. Chem. Soc., 2013, 135, 301.

[46] H.-Y. Chen, M.-H. Lin, C.-Y. Wang, Y.-M. Chang, S. Gwo, J. Am. Chem. Soc., 2015, 137, 13698.

[47] P. B. Johnson, R. W. Christy, Phys. Rev. B, 1972, 6, 4370.

[48] I. H. Malitson, M. J. Dodge, J. Opt. Soc. Am., 1972, 62, 1405.

[49] M. A. Green, M. J. Keevers, Prog. Photovolt: Res. Appl., 1995, 3, 189. 


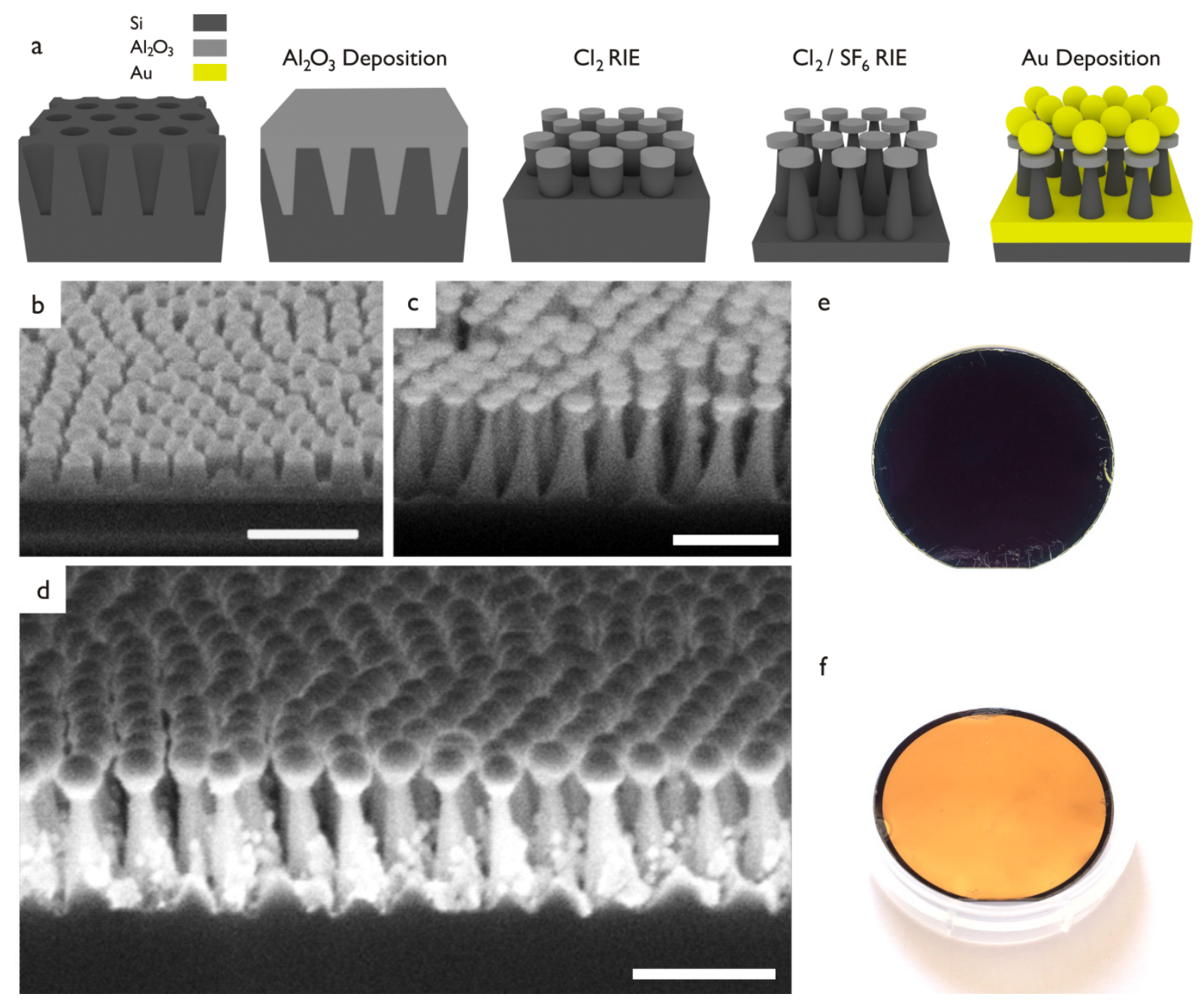

Figure 1. (a) Schematic of the nanofabrication process to produce GNRH SERS substrates.

(b-d) Cross-sectional SEM images of the (b) alumina/silicon nanocylinder arrays after $180 \mathrm{~s}$ of chlorine-based RIE, (c) alumina/silicon nanohoodoo arrays after an additional treatment of 30 s by mixed chlorine- and fluorine-based RIE and (d) the GNRH substrate consisting of arrays of alumina/silicon nanohoodoos topped by a layer of separated gold nanoparticles formed by e-beam evaporation of $40 \mathrm{~nm}$ thick gold. Scale bars: $200 \mathrm{~nm}$. (e) and (f) Photographs of 2-inch wafers covered with arrays of nanohoodoos, taken before and after gold deposition, respectively. 

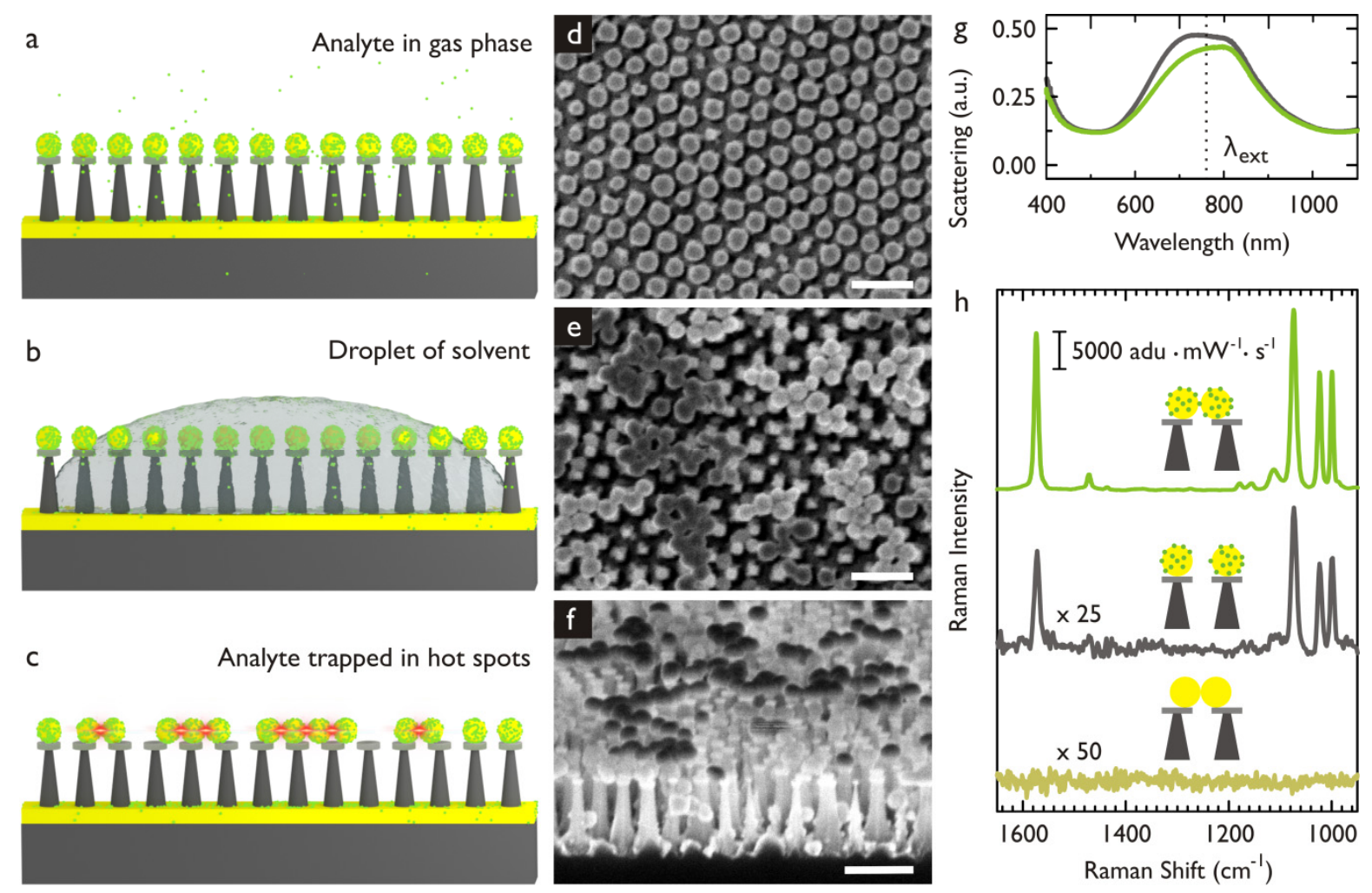

Figure 2. (a) - (c) Concept of gas sensing using the GNRH SERS substrate. Dark grey: silicon; light grey: alumina; yellow: gold; green: analytes. (d) A top-view SEM image of the nanohoodoo arrays covered by a layer of separated gold nanoparticles. (e) and (f) SEM images of the nanohoodoos topped by gold nanoparticles aggregations, which are formed during the evaporation of the solvent. Scale bars: $200 \mathrm{~nm}$. (g) Dark-field scattering spectra of the nanohoodoo arrays topped by separate gold nanoparticles (grey) and gold nanoparticle aggregations (green). (h) SERS background and SERS spectra of thiophenol obtained on the GNRH substrate. The gold nanoparticle aggregations are formed upon drying of DI water. $1 \mathrm{mM}$ thiophenol in ethanol solution is evaporated for $1 \mathrm{~min}$ onto the substrate. SERS system 1 is used for the measurements. For $(\mathrm{d})-(\mathrm{h})$ the deposition thickness of gold is $40 \mathrm{~nm}$, resulting in gold nanoparticles with a radius of $\sim 30 \mathrm{~nm}$; The height of the structures is $\sim 190$ $\mathrm{nm}$, which is defined as the vertical distance from the center of the gold nanoparticle layer to the underlying gold film on the silicon substrate. 

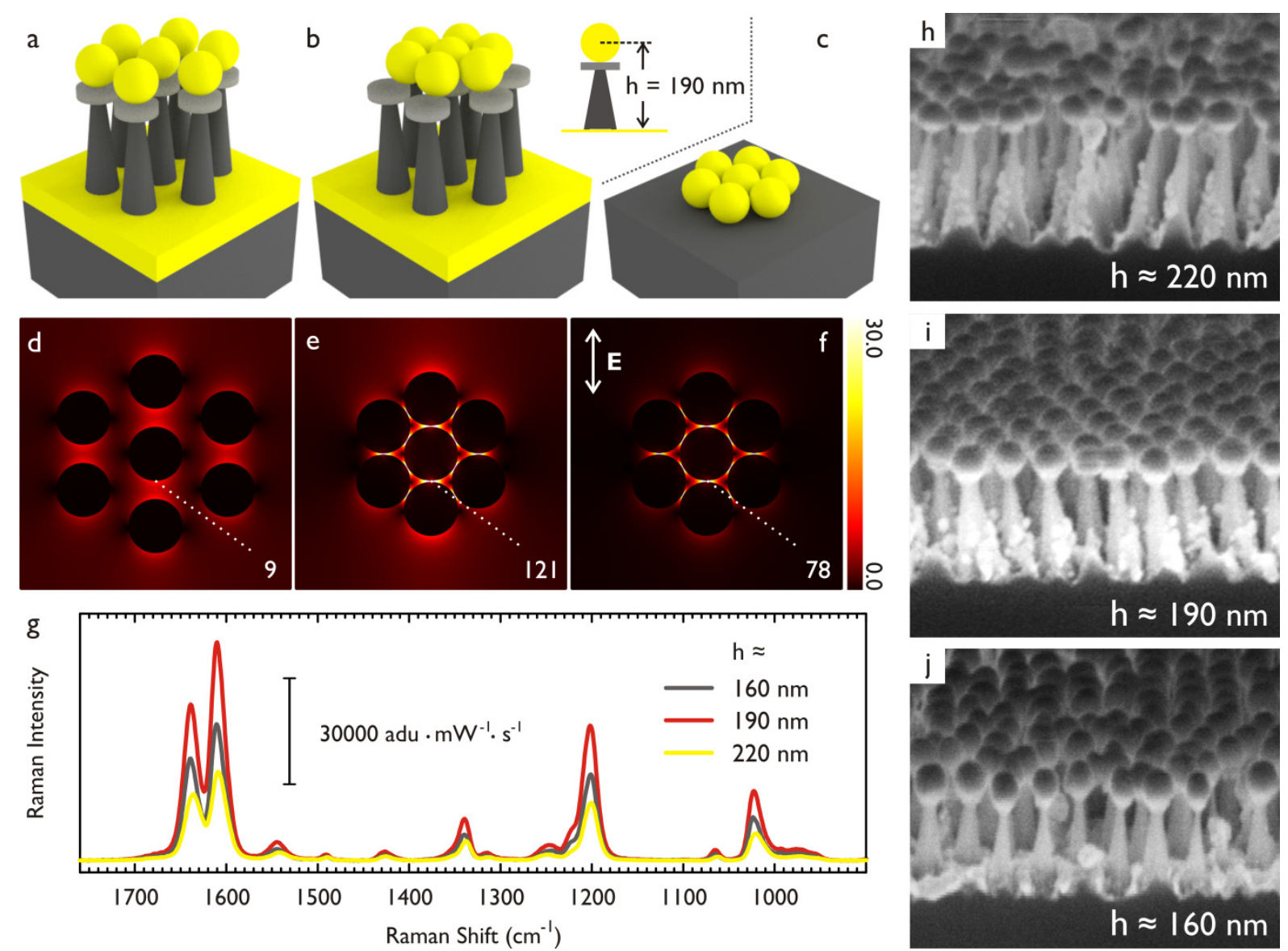

Figure 3. (a) - (c) Simulated 3D structures in (d) - (f), respectively. Dark grey: silicon; light grey: alumina; yellow: gold. (a) A hexagonal array of seven alumina/silicon nanohoodoos each topped by a gold nanoparticle. (b) A hexagonal array of seven alumina/silicon nanohoodoos topped by an aggregation consisting of seven gold nanoparticles. The minimum gap distance between two adjacent gold nanoparticles is $1 \mathrm{~nm}$. (c) The same gold nanoparticle aggregation as in (b) but on a silicon substrate. (d) - (f) Electrical field distributions across the center plane of the nanoparticle layer. The excitation wavelength is $780 \mathrm{~nm}$. (g) SERS spectra of BPE obtained on the GNRH substrate with different heights of $\sim 160, \sim 190$ and $\sim 220 \mathrm{~nm}$. $1 \mathrm{nmol} \mathrm{BPE}$ in ethanol is pipetted onto each substrate. Aggregations of gold nanoparticles are formed during drying of the analyte solution. The measurements are performed using SERS system 1 . In (g), each spectrum is an average of 10 spectra obtained randomly near the centers of the droplet areas. (h) - (j) SEM images of the GNRH substrates used for obtaining the SERS spectra shown in (g). 

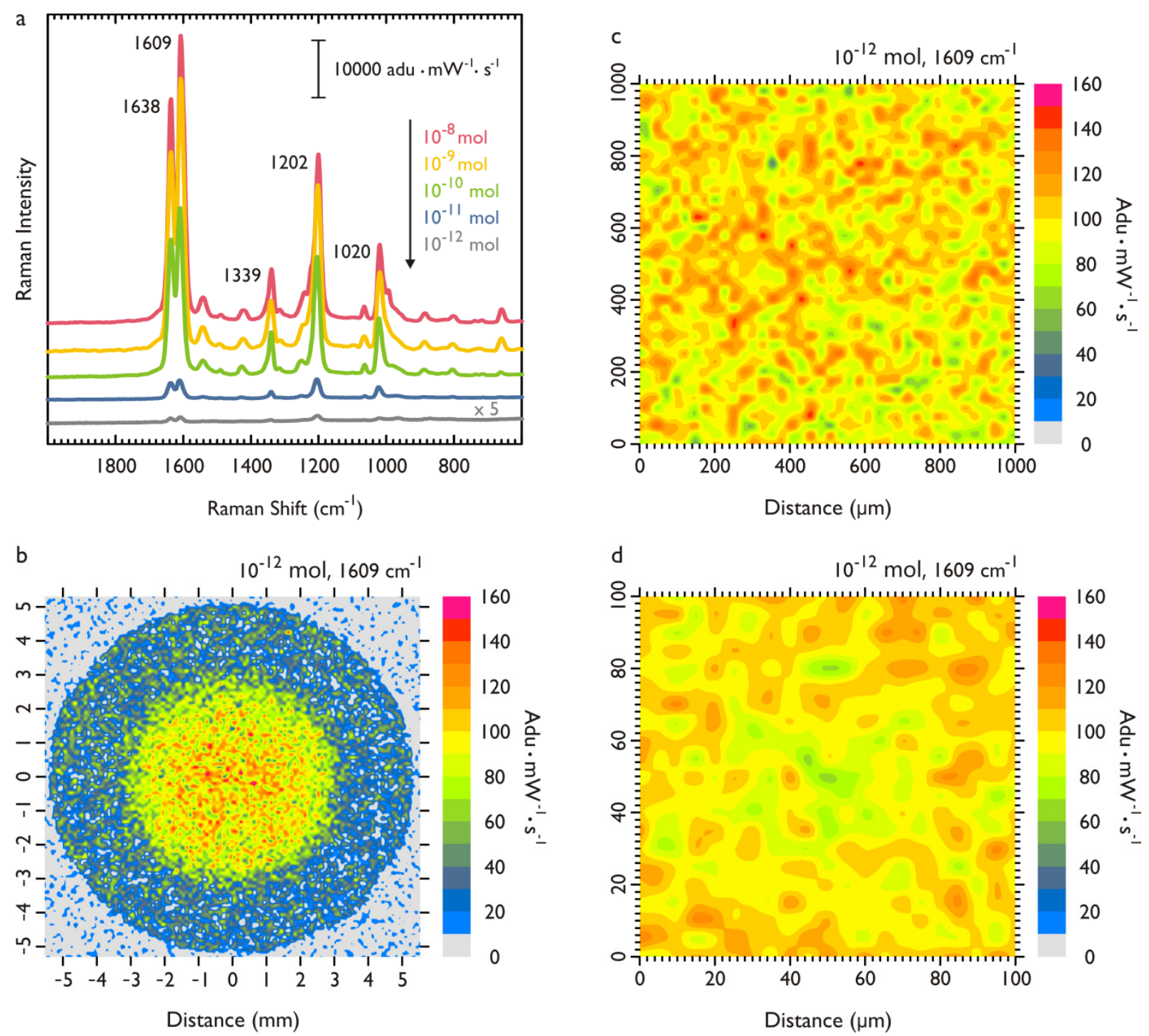

Figure 4. (a) SERS spectra of BPE of different quantities obtained on the GNRH substrates ( $190 \mathrm{~nm}$ in height). Before every measurement, BPE in $1 \mu \mathrm{L}$ ethanol solution is first pipetted onto the substrate. Aggregations of gold nanoparticles are then formed during the drying of analyte solution. (b) - (d) SERS intensities at $1609 \mathrm{~cm}^{-1}$, mapped for the $10^{-12} \mathrm{~mol}$ case in (a), across different areas with different step sizes: (b) the whole droplet area is mapped, with a step size of $100 \mu \mathrm{m}$, (c) a $1 \mathrm{~mm} \times 1 \mathrm{~mm}$ square near the center of the droplet is mapped, with a step size of $10 \mu \mathrm{m}$, and (d) a $100 \mu \mathrm{m} \times 100 \mu \mathrm{m}$ square near the center of the droplet is mapped, with a step size of $5 \mu \mathrm{m}$. All the measurements are performed using SERS system 2 . 

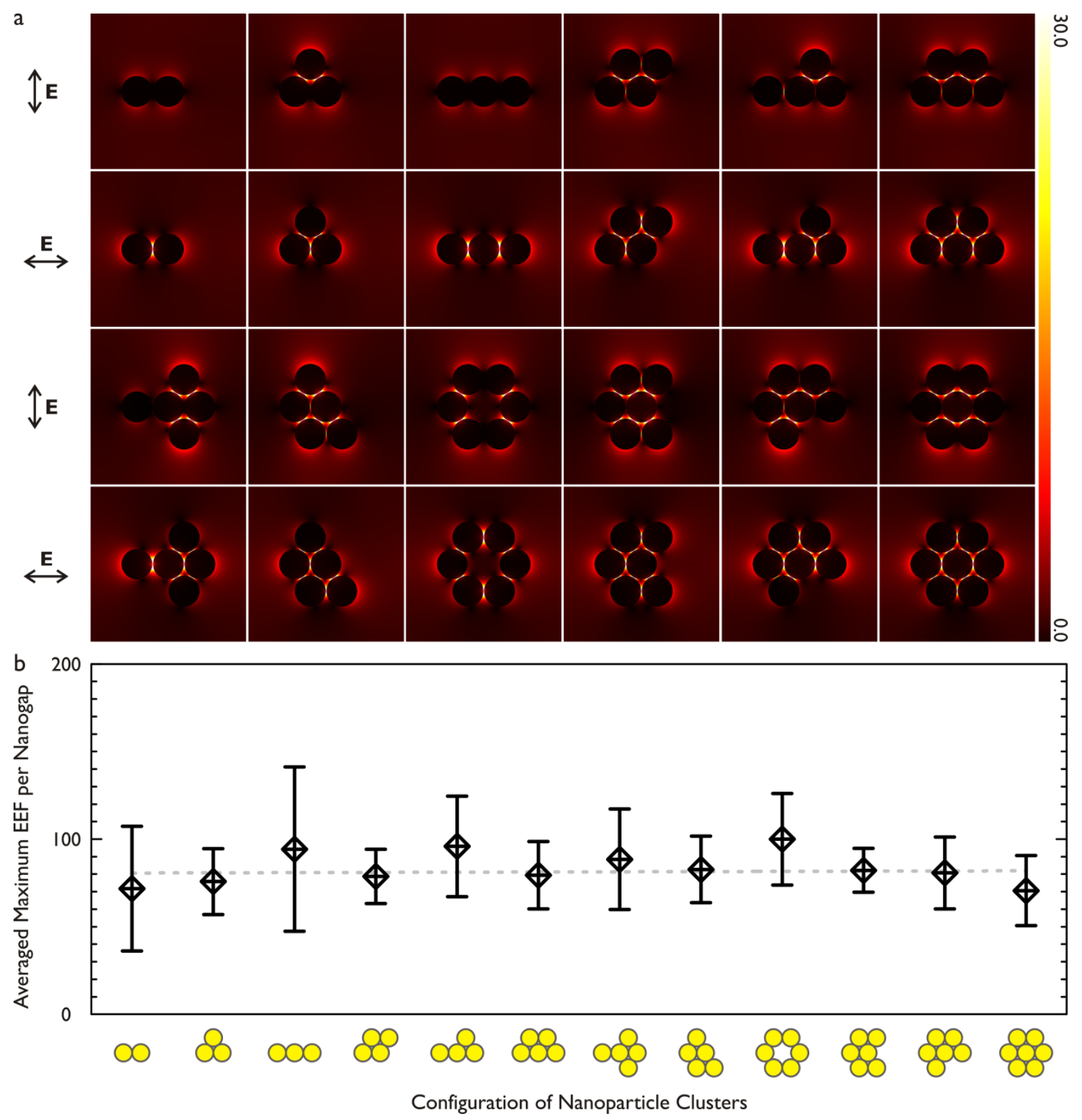

Figure 5. (a) Simulated electrical field distributions across the central plane of the gold nanoparticle layer for the GNRH substrate. The 3D simulation model is the same as is shown in Figure 3a, except for that the nanohoodoos are topped by different clusters of gold nanoparticles. The minimum gap distance between two adjacent gold nanoparticles is $1 \mathrm{~nm}$. The excitation wavelength is $780 \mathrm{~nm}$. (b) The calculated polarization-averaged maximum EEF per nanogap versus the configuration of the nanoparticle clusters. 

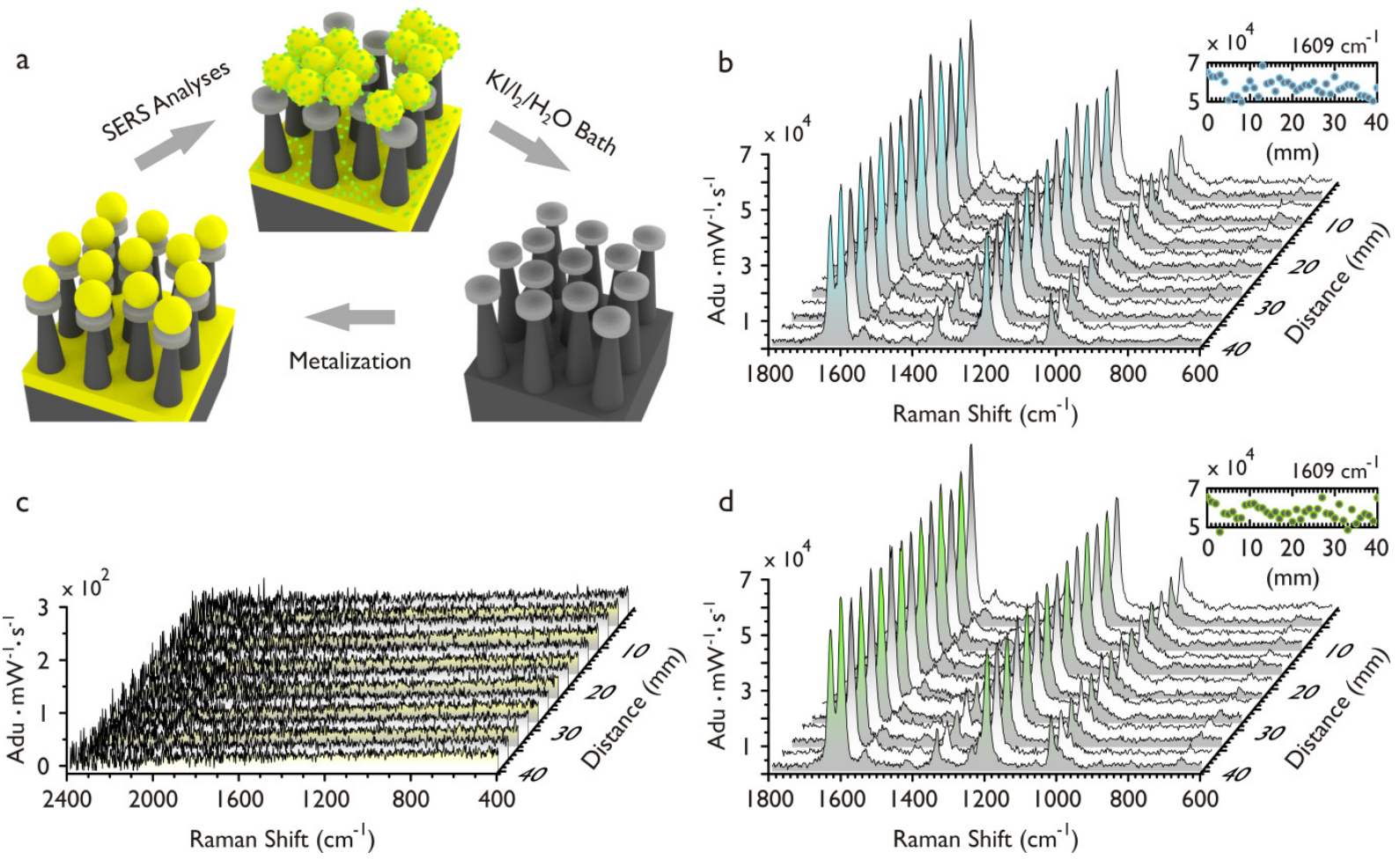

Figure 6. (a) Workflow of recycling the alumina/silicon nanohoodoos. Dark grey: silicon; light grey: alumina; yellow: gold; green: analytes. (b) SERS line scan of BPE obtained on the GNRH substrate. The substrate is incubated in $200 \mu \mathrm{M}$ BPE in ethanol solution for 5 min. Aggregations of gold nanoparticles are formed on top of the nanohoodoos during drying of the solution. (c) Line scan of SERS background obtained on the renovated GNRH substrate, covered with gold nanoparticle aggregations which are formed upon drying of DI water. (d) Same as (b), but a renovated substrate is used. SERS measurements are performed using SERS system 2. 


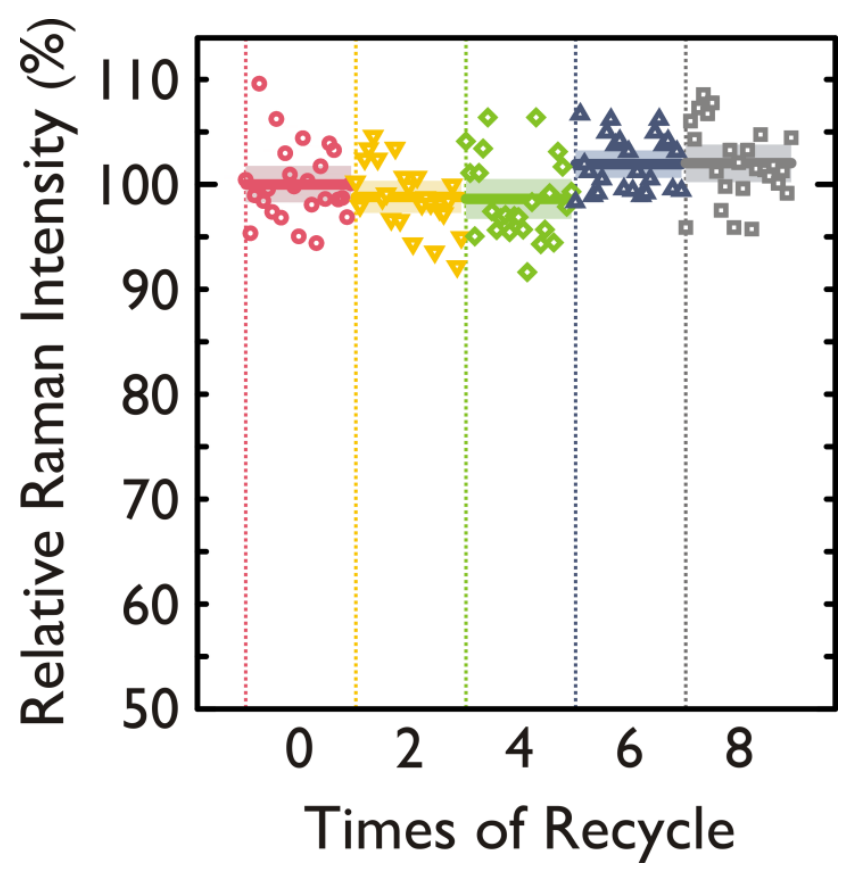

Figure 7. (a) Relative SERS intensities of BPE at $1609 \mathrm{~cm}^{-1}$ obtained on a GNRH substrate over its 8 times of regeneration. For each measurement, $1 \mathrm{nmol}$ BPE in ethanol is first pipetted onto the substrate. Aggregations of gold nanoparticles are then formed during the drying of analyte solution. Afterwards, 25 spectra were obtained using SERS system 2. The average values are shown by the solid lines, while the CVs are represented by the shadow regions. 


\section{The table of contents entry}

Cheap, robust, macroscopically uniform and highly efficient substrates for surface-enhanced Raman spectroscopy (SERS) are fabricated using block copolymer lithography. A nanohoodoo template in the substrate is recyclable after SERS analyses via a simple wet chemistry process. The renovated substrate exhibits reproducibly low SERS backgrounds and identical SERS perfomance, in comparison to a new substrate.

Keyword: surface-enhanced Raman spectroscopy, SERS substrate, block copolymer lithography, recyclable, large-area SERS uniformity

Kaiyu $\mathrm{Wu}^{\dagger}$, Tao $\mathrm{Li}^{\dagger}{ }^{\dagger}$, Michael Stenbæk Schmidt, Tomas Rindzevicius, Anja Boisen* and Sokol Ndoni

${ }^{\dagger}$ Equal contribution

Dr. K. Wu, Assoc. Prof. M. S. Schmidt, Assoc. Prof. T. Rindzevicius, Prof. A. Boisen and Snr. Scientist S. Ndoni

Department of Micro- and Nanotechnology

Technical University of Denmark

Ørsteds Plads, Building 345B, 2800 Kgs. Lyngby

Denmark

Dr. T. Li

Department of Electronic \& Electrical Engineering

University College London

Torrington Place, London WC1E 7JE

UK

Gold Nanoparticles Sliding on Recyclable Nanohoodoos - Engineered for Surfaceenhanced Raman Spectroscopy

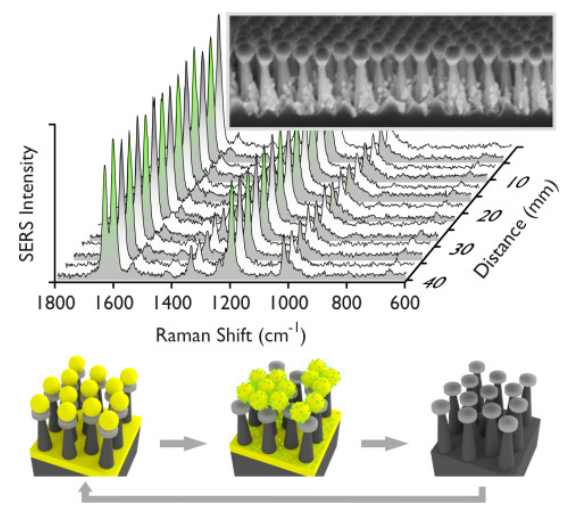


Copyright WILEY-VCH Verlag GmbH \& Co. KGaA, 69469 Weinheim, Germany, 2013.

\section{Supporting Information}

Gold Nanoparticles Sliding on Recyclable Nanohoodoos - Engineered for Surfaceenhanced Raman Spectroscopy

Kaiyu $\mathrm{Wu}^{\dagger}$, Tao Lit,*, Michael Stenbak Schmidt, Tomas Rindzevicius, Anja Boisen* and Sokol Ndoni

${ }^{\dagger}$ Equal contribution

Dr. K. Wu, Assoc. Prof. M. S. Schmidt, Assoc. Prof. T. Rindzevicius, Prof. A. Boisen and Snr. Scientist S. Ndoni

Department of Micro- and Nanotechnology

Technical University of Denmark

Ørsteds Plads, Building 345B, 2800 Kgs. Lyngby

Denmark

Dr. T. Li

Department of Electronic \& Electrical Engineering

University College London

Torrington Place, London WC1E 7JE

UK
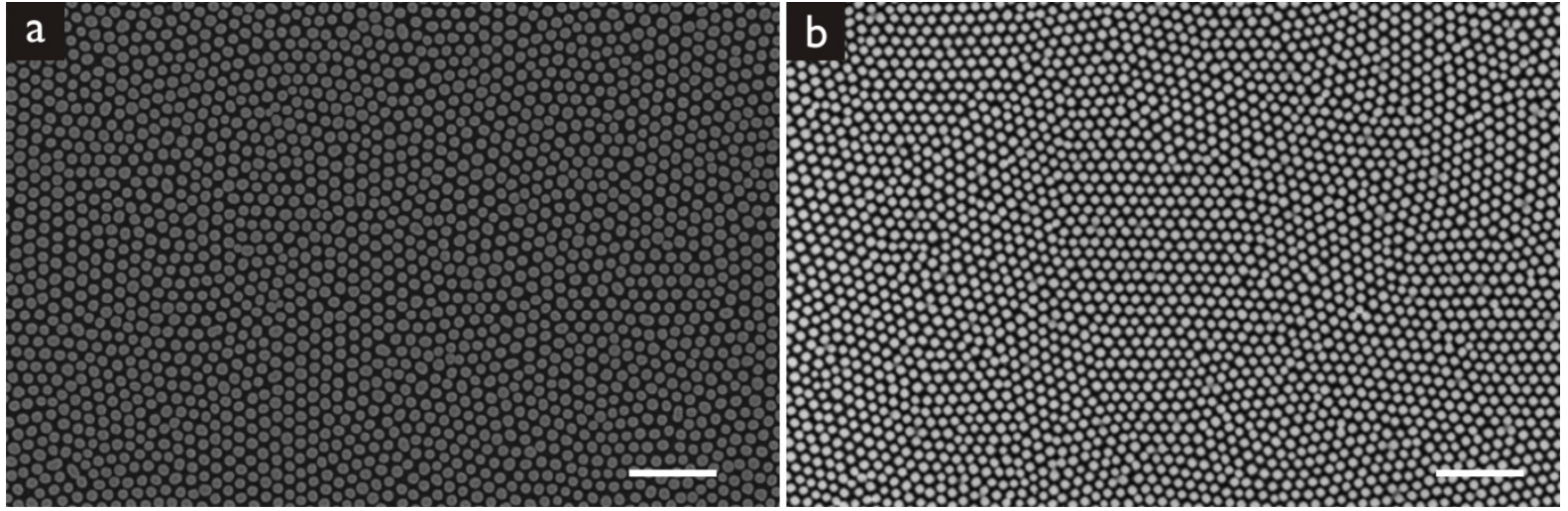

Figure S1. Top-view SEM images of (a) alumina/silicon nanocylinder arrays after $180 \mathrm{~s}$ of

chlorine-based reactive ion etching, and (b) recycled alumina/silicon nanohoodoos. Scale bar: $500 \mathrm{~nm}$. 


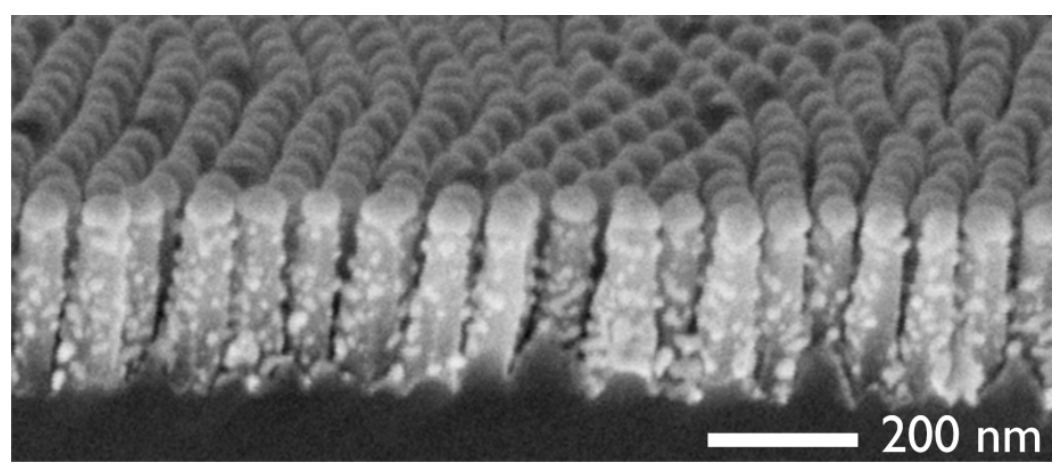

Figure S2. A cross-sectional SEM image of the alumina/silicon nanocylinder arrays covered by gold after deposition and drying of DI water. No fluorine-based reactive ion etching is employed. 
a

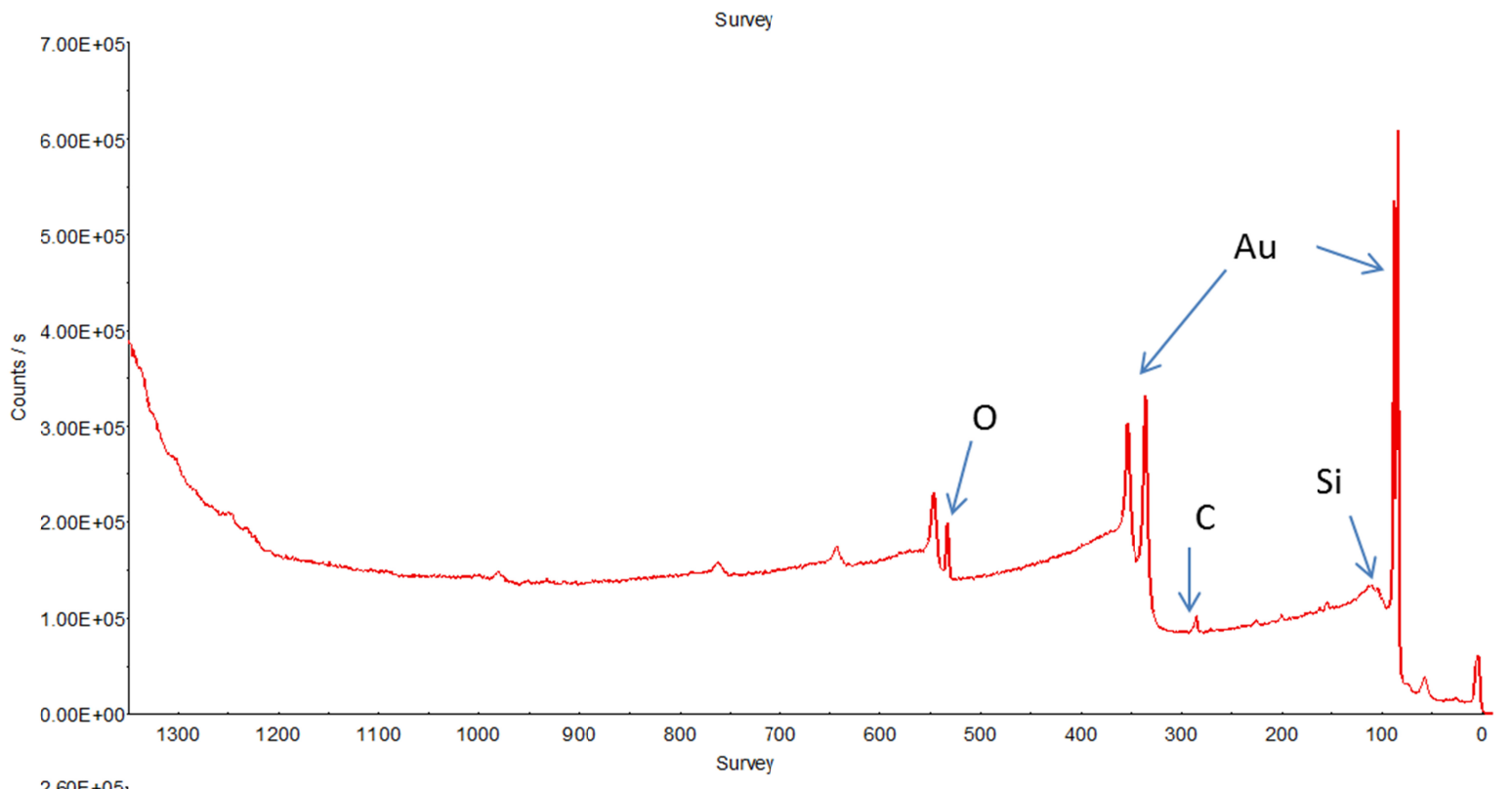

b

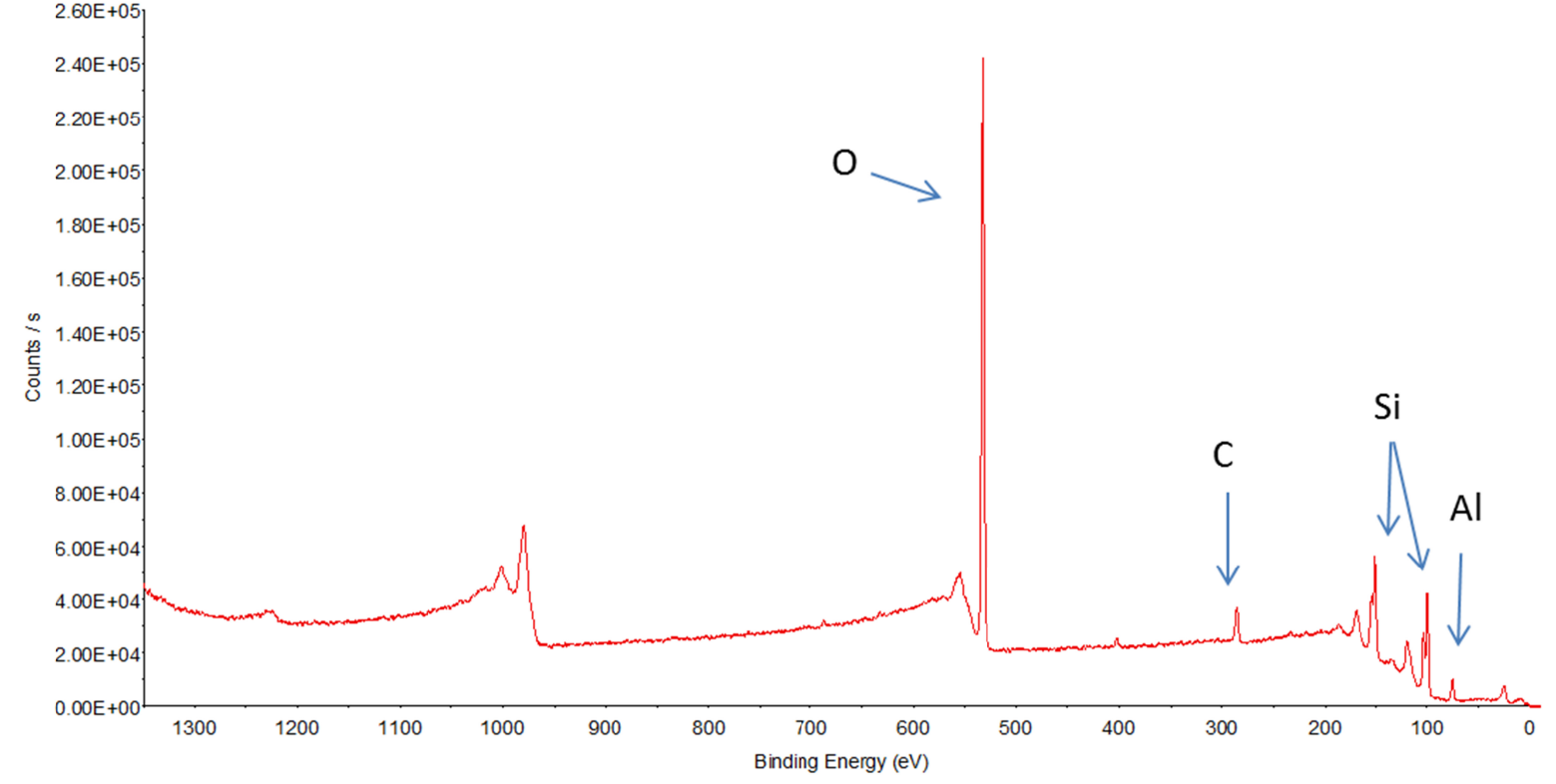

Figure S3. XPS spectra obtained on (a) the SERS substrate containing nanohoodoos topped by gold nanoparticles and (b) the recycled nanohoodoos after removal of gold. 